•综述・

\title{
自然杂交与物种形成
}

\author{
王玉国 ${ }^{1,2 *}$ \\ 1 (生物多样性与生态工程教育部重点实验室, 复旦大学生物多样性科学研究所, 上海 200438) \\ 2 (复旦大学生命科学学院生态与进化生物学系, 上海 200438)
}

\begin{abstract}
摘要: 在生物进化过程中, 自然杂交对物种形成有着重要的影响。多倍化和同倍体杂交物种形成是通过杂交产生 新种的两种主要方式。普遍的观点认为多倍化是植物物种形成的主要动力，而越来越多染色体倍性相同物种之间 杂交现象的发现, 说明同倍体杂交也是物种形成的重要方式。目前按严格标准确定的同倍体物种形成案例相对较 少, 还亟需对更多不同类型的种间杂交带进行形态与分子等多方面证据的调研, 并通过模型预测与实测数据分析, 来探讨杂交物种形成初期乃至整个过程的遗传基础; 更多杂交物种形成的新模式材料, 有待于通过分化谱系的历 史基因流重建进行鉴定，用于进一步阐释自然选择对于生殖隔离形成的影响、物种形成过程中的生态适应变化和 多样性遗传创新的产生规律。本文简要综述了自然杂交的研究历史，阐述其相关概念、研究方法和基因组时代杂交 与物种形成研究的最近进展, 并针对拟解决的关键问题提出相应的分析见解, 以期为后续研究和涉及杂交的物种多 样性保护提供借鉴。
\end{abstract}

关键词: 自然杂交; 物种形成; 多倍化; 同倍体杂交; 渐渗杂交

\section{Natural hybridization and speciation}

\author{
Yuguo Wang ${ }^{1,2^{*}}$ \\ 1 Ministry of Education Key Laboratory for Biodiversity Science and Ecological Engineering; Institute of Biodiversity \\ Science, Fudan University, Shanghai 200438 \\ 2 Department of Ecology and Evolutionary Biology, School of Life Sciences, Fudan University, Shanghai 200438
}

\begin{abstract}
Natural hybridization plays a pivotal role in the formation of new species during the evolution of organisms. There are two principal types of hybrid speciation: polyploidization and homoploid hybrid speciation. The former has been regarded as an important force driving plant speciation, whereas the latter has proved to be a main mode of speciation based on an increasing number of cases, which have reported successful crosses between the species at the same ploidy level. However, only a few cases of homoploid hybrid speciation have been documented when strict criteria are applied. Therefore, molecular evidence involving more genomic loci and morphological investigations from different kinds of hybrid zones, as well as assessments of existing speciation models and new computer stimulations, are required for further understanding the genetic basis of the initial and entire process of speciation. Through the historical reconstruction of gene flow between diverging lineages, additional organismal models for hybrid speciation need to be developed to reveal the effects of natural selection on the formation of reproductive isolation, and to discern the ecologically adaptive changes and the formation rules of novel diversity in the process of hybrid speciation. Here I briefly review the history of studies examining natural hybridization and speciation to introduce concept changes, research methods, and the latest advances of natural hybridization and speciation, to identify the unsolved core and basic scientific questions and to provide feasible suggestions for future studies and the protection of biodiversity involved in natural hybridization.
\end{abstract}

Key words: natural hybridization; speciation; polyploidization; homoploid hybrid speciation; introgressive hybridization 
自然杂交(natural hybridization)是在自然条件 下，来自基于某一个或多个可遗传的性状可以明显 区分的两个或多个不同群体的个体之间的成功杂 交(Arnold，1997)。在生物长期进化的过程中, 自然 杂交与物种形成(speciation)存在着重要的关联。由 于自然杂交的客观存在, 杂交后代与亲本之间的系 统发育关系从本质上就构成了与“二叉分歧”结构不 同的网状形式, 这种进化就是网状进化 (reticulate evolution)。因此而导致产生新物种的方式就是杂交 物种形成(hybrid speciation)。

对于杂交与物种形成关系的认识, 在动植物的 研究中存在明显不同的见解。植物类群中自然杂交 的大量出现, 表明新的杂交种和新的适应型对进化 产生了显著影响。然而, 在动物方面的自然杂交研 究却是普遍支持另一个截然不同的观点: 由于种间 杂交几乎不产生或很少产生可育的后代, 是不适应 的, 因而被看作是一个导致生殖隔离形成的过程。 自然杂交究竟是一个潜在的创造过程(Lotsy, 1916; Anderson \& Stebbins, 1954; Lewontin \& Birch, 1966; Arnold, 1992), 有助于物种分化 (Rieseberg \& Wendel, 1993; Arnold, 1997), 还是进化过程中的“噪 音(noise)”或“盲端(dead end)” (Wagner, 1970; Mayr, 1992; Schemske, 2000), 曾存在广泛的争议。随着基 因组时代的到来, 很多未决的问题获得了突破, 更 多的研究已从关注自然杂交的鉴定, 聚焦到杂交物 种形成的过程和相关的机制上来。

\section{1 种间杂交的方式与物种形成}

自然杂交的遗传本质是分化谱系间遗传物质 的交流, 在后代中发生重组、变异以及频率的变化 并导致性状改变, 是基因组进化和物种形成的主要 动力之一。虽然在动植物中均有案例显示种间杂交 后代可能在适合度(fitness)上相比亲本处于劣势, 后代育性低或不育, 因而会被淘汰, 但也有大量研 究表明, 可存活的种间杂交后代能够综合亲本的适 应性或创造出新的适应性, 丰富基因库、拓宽生境, 进而促进基因组进化和新种形成。

根据遗传物质交流时是否发生染色体数加倍 以及与亲本之间是否产生生殖隔离, 可将种间杂交 划分为 3 种主要进化方式: 多倍化 (polyploidization)、同倍体杂交物种形成 (homoploid hybrid speciation)和渐渗杂交(introgressive hybridization)。

\section{1 多倍化}

多倍化是被普遍认可的植物最主要的物种形 成方式(Abbott, 2003)。来自不同物种的配对染色体 在减数分裂时通常无法正常分离, 导致杂交后代不 育。但在多倍体中, 通过染色体加倍, 每个染色体 对在减数分裂时包含来自一个物种的两个染色体, 正常的分离就可以得到保证，从而达到后代杂交可 育。由于杂交后代和亲本的染色体数目不匹配，从 而使其与亲本之间产生生殖隔离。因此, 可以相对 容易地被确定是新的物种。在一些植物中, 异源多 倍化(allopolyploidization)甚至被公认为能“瞬间”生 成新种，例如，四倍体邱园报春(Primula kewensis) 是由两个二倍体物种轮叶报春 (P. verticillata) 和多 花报春(P. floribunda)杂交而来(Ramsey \& Schemske, 2002)。

\section{2 同倍体杂交物种形成}

染色体倍性相同的物种之间也可以通过杂交 产生新的物种。这种方式通常表现为二倍体物种之 间杂交后形成新种，如向日葵属(Helianthus)、药药 属(Paeonia)等类群中都有这类种间杂交。这些倍性 与亲本相同的杂交后代可以通过基因重组产生与 双亲不同的遗传组合，因此也称同倍体重组 (homoploid recombination)。其他倍性相同物种之间 (如四倍体与四倍体、六倍体与六倍体)的杂交也属 于这个范畴(Ferguson \& Sang, 2001)。确定同倍体种 间杂交后代与亲本之间是否存在生殖隔离, 是判断 新种形成的关键和难点, 往往需要对两个亲本种相 互接触的地带——杂交带(hybrid zone, 或译作杂交 区、杂交地带)群体开展大量的形态和分子遗传学研 究。来自不同亲本的基因型通过杂交会在此区域形 成遗传上由一个亲本向另一个亲本过渡的渐变群 (cline)。在杂交背景上, 两个亲本类型形成了斑块化 分布的镶嵌式结构。杂交带宽度从几十米到几百公 里不等, 其形状差异取决于杂交个体的存活比率高 低。对杂交带精确定义的理解, 不同研究者的侧重 有所不同: 有的强调该区域杂交个体适合度的增加; 有的则强调杂交后代是亲本之间二次接触的结果, 尽管杂交后代和亲本基因流可能依然存在，但两者 能够通过表型差异和特定遗传位点来进行区分。

\section{3 渐渗杂交}

杂交后代可与两个亲本物种或其中之一反复 回交, 结果会导致从一个种到另一个种跨越生殖障 
碍的基因转移，这种自然杂交方式即渐渗杂交 (Anderson \& Hubricht, 1938; Anderson, 1949)。在自 然条件下, 动植物发生渐渗杂交的例子不胜枚举。 典型的如狼 [ 野狼 (wolf, Catrans lupus) 和灰狼 (coyote, C. latrans)]、鲤科骨尾鱼属(Gila)等动物; 煘 尾属(Iris)、刺柏属(Juniperus)、松属(Pinus)和禾本 科一些属等植物。如果杂交后代超过 $\mathrm{F}_{1}$ 代、并且存 在杂交个体之间的交配以及与亲本的回交, 这样的 群体就是杂交群(hybrid swarm)。这个概念很容易与 杂交带的渐变群混淆。杂交群高度可变, 由介于两 个亲本类型之间的遗传和表型特征存在差异的一 系列个体组成, 因此杂交群使得亲本类群之间的界 限变得模糊。例如, 目前野生稻(Oryza rufipogon)的 生境比较适合栽培稻( $(O$. sativa $)$ 的生长, 如果没有 有效的隔离, 野生稻很可能就会变成被栽培稻基因 普遍渐渗的类型。当杂交个体存在变异、与亲本类 型一样有活力, 并且与亲本之间的回交不存在障碍, 杂交群就发生了。但如果杂交类型很少变异, 或者 杂交群个体不与亲本类型回交就能维持, 杂交群则 不能发生。渐渗杂交一般不能产生新的物种, 但如 果这些可育的种间杂交不再与亲本群体发生回交 或占据了新生境, 就可能形成新的杂交种(Abbot, 2003)。

\section{2 自然杂交的频率}

总的来说，在动物中发生种间自然杂交事件的 频率非常低, 而在植物中则相对普遍 (通常分别用 rare和widespread来形容, Arnold, 1997)。尽管如此, 植物种间杂交的整体发生频率并不高, 仅在某些特 定的分类群尤其是近缘分类群之间有很高的杂交 频率, 显示出明显的偏好, 比如: 松科、桦木科、柳 叶菜科、蓄薇科、杨柳科等类群中杂交事件发生频 繁, 但在唇形科和豆科中却很少发生。对不同区系 的维管植物统计发现: 每一个区系中种间杂交在 科、属的水平出现频率都是不均等的(Ellstrand et al, 1996)。此外, 多年生植物发生杂交的频率往往高于 一年生植物(Stace，1975)。但也有学者估计平均有 $10 \%$ 的植物和 $25 \%$ 的动物至少和另一个物种发生过 杂交(Mallet, 2005), 在快速辐射进化类群的近缘物 种间更容易发生杂交。自然杂交事件在不同分类群 中不均等地出现, 一方面反映了生物体自身的差异 (如交配系统的差异), 另一方面与其时空分布有着
密切关系, 如同域分布类群与异域分布类群在自然 杂交方面就可能面临不同的机遇。

Vriesendorp和Bakker (2005)统计了已发表文献 中涉及被子植物不同种间杂交方式的进化案例, 发 现多倍化现象比同倍体重组更为普遍。Kim 和 Lockhart (2006)则认为同倍体杂交物种的形成不应 被看作是罕见的现象, 由于同倍体重组不像多倍化 现象容易鉴定, 很可能被严重低估, 因为很难将它 和一般的杂交与渐渗杂交相区分。

一些研究表明, 大多数被子植物起源于祖先的 杂交(Clausen et al, 1945; Stebbins, 1947; Soltis \& Soltis，1993)。但也有研究推测只有大约 2-4\%的被 子植物和 $7 \%$ 的烣类植物为多倍体杂交起源(Otto \& Whitton, 2000), 细胞学、化石和基因组的证据支持 绝大多数被子植物和屏类为古多倍体 (paleopolyploidy), 存在祖先类群的基因组加倍(Grant, 1981; Masterson, 1994; Cui et al, 2006)。Wood等(2009)通 过对细胞学资料和物种间系统发育关系的联合分 析，确定约有 $15 \%$ 的被子植物和 $31 \%$ 的硕类植物的 物种形成事件中经历了染色体倍性增加, 但加倍后 并没有必然导致相关谱系分化速率的加快。

\section{3 鉴定自然杂交的通用方法}

\section{1 方法概述}

早期对杂交的鉴定主要依据形态特征, 杂交指 数常被用于区分双亲间的中间类型并推断个体是 否来自杂交。但形态特征的可塑性常使子代和双亲 混淆。由于形态标记的可靠性较低, 没有一致的标 准, 甚至不同的研究者都可能获得截然不同的判定 结果, 因此仅依靠形态标记来鉴定杂交很容易导致 错误的判断。细胞学标记曾作为杂交的重要判断标 准，同倍体杂交物种形成和多倍化的确定都依靠染 色体数目的准确鉴定, 但实际操作时必须明确染色 体的倍性和亲本范围, 因而也存在很大的局限性。

分子标记在自然杂交的鉴定与分析中曾发挥 重要的作用。它包括可遗传并可检测的特异性蛋白 质标记和核酸标记。蛋白质标记方法(如同工酶和等 位酶等)因精确度明显不足, 逐渐被后来发展的 DNA标记取代。常用的DNA标记主要有限制性长度 片段多态性(restriction fragment length polymorphisms, RFLP)、随机扩增多态性DNA (random amplified polymorphic DNA，RAPD)、扩增片段长度多态性 
(amplified fragment length polymorphisms, AFLP)、 简单重复序列(simple sequence repeats, SSR或称微 卫星)等, 其中RFLP、SSR为共显性标记, 能鉴定种 间杂交及亲本, 应用最为广泛。这些分子标记是针 对整个基因组的扩增, 检测效率高、信息量大, 但所 获得的信息由扩增片段的有无决定, 受实验条件影 响较大, 鉴定结果不如基因序列准确。

基因序列的系统发育分析, 是研究自然杂交尤 其是种间杂交最为常用的方法, 也是鉴别手段的首 选(Arnold, 1997)。鉴定种间杂交时, 首先要选择适 于属内种间甚至是更低分类群的基因, 并确保其对 近缘类群有足够的分辨率。植物基因组包括核基因 组、叶绿体基因组和线粒体基因组, 绝大多数动物 无叶绿体基因组。了解不同基因组的特点以及属内 种间甚至群体间研究的基因或DNA片段, 将有助于 合理而有效地应用它们去获得系统发育信息, 判断 自然杂交。

叶绿体基因组和线粒体基因组通常为单亲遗 传, 绝大多数为母性遗传, 因此常被用来判断杂交 种的母本。植物叶绿体基因组中编码基因中的 $m a t K$ 基因、非编码序列(内含子或间隔区)中的 $t r n L-F$ 、 $r p l 16$ 、trnT-L、trnG-S、trnC-D、rps16等都曾被广 泛用于不同属内种间的物种鉴定, 适于种间杂交和 亲本的判定, 而线粒体基因仅有 nad1和nad4等少数 有稳定变异的长内含子基因片段被应用于植物种 间杂交的进化分析(Chat et al, 2004)。目前大量植物 叶绿体基因组已经被报道, 富含信息位点的长基因 片段篎选及相关引物设计比较容易; 而线粒体组基 因重排事件频繁, 很多类群还缺乏相关数据。相比 较而言, 动物的线粒体基因组排列相对稳定, 常用 于属内种间系统发育的基因有细胞色素氧化酶I (cytochrome c oxidase subunit I, COI)、细胞色素 $\mathrm{B}$ (cytochrome b subunit, cytB)、16S rDNA等基因。 由于动物线粒体基因组相对较小, 也适合用完整线 粒体组来确定争议物种的界限问题, 如虎鲸的分类 (Morin et al, 2010)。

核基因组为双亲遗传，常被用来判断双亲的大 致范围, 可避免仅凭单亲遗传推断而产生的不正确 分支关系解释。虽然核基因组包含的基因的数量和 变异相当大, 但以往用于植物属内种间杂交的核基 因序列最多的却是核糖体DNA (nrDNA) ITS。它由 介于 $18 \mathrm{~S}$ rRNA、 $5.8 \mathrm{~S} 、 26 \mathrm{~S}$ rRNA之间的两段序列
(ITS1和ITS2)组成。其突出的优点包括: 结构功能 十分保守, 但又具有一定的变异; 在细胞中含量大, 容易获得。虽然ITS在基因组中存在大量拷贝，但在 多数植物中为一致性进化(concerted evolution, Elder \& Turner, 1995), 多数情况下可以看作是单拷贝基 因, 因而成为进行属内种间系统发育研究和自然杂 交判断的理想片段, 得到广泛应用。由于种间杂交 后代特别是 $F_{1}$ 代会包含来自双亲物种的序列, 而大 部分核基因中同时存在直系同源和旁系同源拷贝， 因此使用核基因进行杂交起源判断时必须进行分 析和鉴别。除核糖体DNA以外，一些含有长内含子 的单或低拷贝核基因有足够的信息位点, 已被陆续 应用于物种间的系统发育研究，这些基因的克隆测 序可以帮助判断自然杂交、多倍化及检验相关的物 种形成假说。目前已成功应用的基因包括Adh (编码 乙醇脱氢酶, Sang et al, 1997; Ferguson \& Sang, 2001)、RPB2 (编码RNA聚合酶II的第二大亚基)、 P giC (编码磷酸葡萄糖异构酶, 一种糖分解酶) 、 PhyC (光敏色素基因家族的一个成员, 编码感光蛋 白) (Russell et al, 2010)、PI (调控花器官发生的B类 基因, Lee et al, 2002)、LEAFY (调控花分生组织和花 期等功能, Kim et al, 2008)、GBSSI (编码小粒结合淀 粉合成酶, Smedmark et al, 2003)等。目前大量的转 录组或基因组序列的测定, 使得从大量功能基因中 篮选出适于属内种间系统发育研究的单拷贝核基 因(single copy nuclear gene, SCNG)更为便利, 特别 是那些含有长内含子的单拷贝核基因最为适合自 然杂交的判定和物种形成假说的验证。

利用不同类型基因的系统发育不一致(phylogenetic incongruence)来判断种间杂交, 是应用比较 普遍的方法, 如Chat等(2004)基于叶绿体基因序列 和线粒体分型对猕猴桃属(Actinidia)网状进化的研 究。在植物自然杂交与进化的研究中, 基于ITS和特 定叶绿体基因的系统发育不一致来判断种间杂交 事件的发生是最为常用的方法, 研究者往往依据叶 绿体基因母性遗传特点来判定杂交种的母本。需要 注意的是, 除了种间杂交, 基因水平转移(horizontal gene transfer)、基因复制后的拷贝丢失(hidden paralogs) 及不完全谱系分选 (incomplete lineage sorting)等过程均可能导致系统树的冲突(Wendel \& Doyle, 1998), 在判断种间杂交与物种形成时应加 以区分。 


\section{2 典型案例}

基于上述通用方法, 从无脊椎动物到脊椎动物, 有大量的渐渗杂交案例被报道(Arnold, 1997)。在果 蝇、Heliconius 属蝴蝶中还有同倍体杂交物种形成被 证实(Schwarz et al, 2005; Mavárez et al, 2006; Melo et al, 2009; Salazar et al, 2010)。

与动物网状进化以渐渗杂交为主不同, 植物自 然杂交的方式更为多样化, 比较有代表性的案例很 多, 如棉属(Gossypium) $\mathrm{AD}$ 基因组的异源多倍化起 源(Wendel et al, 1995)、向日葵属的同倍体古杂交 (Rieseberg et al, 1990; Rieseberg 1991)、药药属的复 杂网状进化(Sang et al, 1995)、感尾属的渐渗杂交 (Arnold et al, 1990a, b, 1991; Arnold, 1993)、具有细 胞质DNA单亲互补遗传模式(Mogensen, 1996)的松 科植物的自然杂交(Isoda et al, 2000; Wang et al, 2001)等。此外, 还有基于单拷贝或低拷贝核基因探 讨异源多倍体物种网状进化重建的研究, 已用于十 字花科独行菜属(Lepidium)、蓄薇科Geinae类植物、 蓼科春蓼属(Persicaria)、兰科多穗兰属(Polystachya) 等多个类群。这种重建方法不仅可以借助基因拷贝 数与植物倍性相关性来探讨杂交起源, 区分同源和 异源多倍体, 还可以推导重要鉴别性状(如花部结 构简化)的进化历史(Lee et al, 2002; Smedmark et al, 2003; Kim et al, 2008; Russell et al, 2010)。

\section{4 基因组时代新的技术方法在自然杂交与 物种形成研究上的应用}

随着新一代基因组测序方法的不断发展，越来 越多的新技术被引入到自然杂交与物种形成的研 究中, 包括: (1) 限制性位点关联DNA测序 (restriction-site associated DNA sequencing, 简称RADseq), 作为一种通用的简化基因组测序(reduced-representation sequencing) 方法, 它已逐渐取代原来的 AFLP、RFLP、SSR等以条带分析的分子标记，在不 同动植物类群杂交与物种形成的研究中获得应用, 例如, 蝴蝶的种间杂交和适应性分化 (Gompert et al, 2012; Nadeau et al, 2013)、丽鱼的杂交物种形成与快 速辐射(Keller et al, 2013; Martin et al, 2015)、蛙类的 渐渗杂交(Streicher et al, 2014)、蚊子性染色体倒位 与生殖隔离 $\left(\mathrm{O}^{\prime}\right.$ Loughlin et al, 2014)等; 再如捕蝇草 属(Ficedula, Rheindt et al, 2014)、马先蒿属(Pedicularis, Eaton \& Ree, 2013)、杨属(Populus, Stölting et al, 2013)、向日葵属(Andrew et al, 2013; Andrew \& Rieseberg, 2013)等植物类群的种间渐渗杂交研究; (2)单核苷酸多态性微阵列(SNP array)技术, 成功用 于在家鼠(Mus musculus domesticus)与近缘类群杂 交带、杨属植物种间杂交带的分析中(Wang et al, 2011; Janoušek et al, 2012, 2015; Geraldes et al, 2014; Liu et al, 2015); (3)外显子组测序 (exome sequencing), Good 等 (2015) 用该方法在两种花栗鼠 (chipmunk)中探讨了生殖隔离与种间杂交的关系; (4)转录组测序(transcriptome sequencing), 该方法已 在很多类群中得到应用, 如家鼠与近缘类群(PhiferRixey et al, 2014)、欧洲野兔 2 个亚种(Oryctolagus cuniculus algirus和O. c. cuniculus, Carneiro et al, 2014)、向日葵属不同物种(Renaut et al, 2013; Barb et al, 2014)等; (5)测序基因分型(genotype by sequencing)技术，在侏儒鸟(manakins)和剑尾鱼(swordtail fish)的案例中, 采用该方法分别揭示了受选择驱动 和遗传不亲和导致的种间杂交与亲本种生殖隔离 (Parchman et al, 2013; Schumer et al, 2014a); (6)全基 因组测序 (whole-genome sequencing), 这种研究最 为详尽的方法目前已成功应用于蝴蝶 (Heliconius Genome Consortium, 2012; Martin et al, 2013)、果蝇 (Garrigan et al, 2012; Lohse et al, 2015)、马(Jónsson et al, 2014)、蚊子(Fontaine et al, 2015)、捕蝇草属 (Ellegren et al, 2012; Nadachowska-Brzyska et al, 2013; Burri et al, 2015)等动植物类群杂交与物种形 成研究的各个方面, 以及尼安德特人、丹尼索瓦人 与现代人之间的基因渗入和遗传重组研究(Green et al, 2010; Sankararaman et al, 2012, 2014; Wall et al, 2013; Lohse \& Frantz, 2014; Prüfer et al, 2014)。纵观 以往的研究方法, 其实不外乎两类: 基于序列分析 的系统发育方法, 基于杂交群或杂交带渐变群等位 基因频率变化的群体遗传学方法。

除此之外，建模分析在杂交物种形成特别是同 倍体物种形成的研究中也得到了发展。早期模型多 属于“适应性基因重组模型”, 主要针对特定杂种基 因型发生正选择或者自交的情况进行的模拟 (McCarthy et al, 1995)。例如, 来自向日葵自然杂交 的建模分析显示，种间杂交中的新基因组合(如倒 位)在经历正选择或自交频率高时就会被固定下来。 这一模型的后续版本整合了种间杂交和亲本的生 态分化, 揭示出如果杂交后代比亲本有更高的适合 
度，杂交物种形成就会频繁出现(Buerkle et al, 2000; Duenez-Guzman et al, 2009)。由于杂交通常会产生 新的性状, 但很难评价这些性状在生态上或进化上 就一定比亲本有更好的适合度, 因此, 最近有研究 者针对上述情况, 提出另一类模型——“遗传不亲 和模型”, 它并不需要考虑是否自交或者是否杂种 相对亲本有适合度优势。计算机模拟结果揭示种间 杂交可以通过对与亲本不亲和的位点进行选择来 实现生殖隔离(Schumer et al, 2015)。值得注意的是, 研究者强调这一模型与以前的模型并不排斥, 但还 需要更多实测案例(如Hermansen et al, 2014)来验证 两者是否兼容。近期在非洲丽鱼中证实古杂交可以 促进快速物种辐射(Meier et al, 2017), 从实测的分 析结果推测, 物种形成所需代数相较以往模型所推 导的会更少，因此，有必要在同一类种间杂交中同 时检验这两类不同模型, 并结合实测数据进行分析 验证。

\section{5 生殖隔离与杂交种的判断标准}

种间杂交并不一定产生杂交种(hybrid species); 但杂交种必然经历了杂交过程。需要强调的是, 在 很多情况下鉴定种间杂交容易, 但要确定已经形成 杂交种——达到物种形成的程度比较困难。这不仅 仅是因为后者确实对于生物的进化有着特殊的意 义, 还因为物种形成本身需要有严格的限定。 Schumer等(2014b)提出了证明自然杂交导致了物种 形成应该满足3个条件: (1)发生了种间杂交; (2)种间 杂交与亲本之间存在生殖隔离; (3)种间杂交导致了 这种生殖隔离。就这些条件而言, 异源多倍化现象 相比同倍体杂交物种形成更容易得到证明。在动植 物自然杂交的案例中大多只证明了(1)或(2)。按 Schumer等(2014b)的分析, 同时满足上述 3 个严格 的标准能够被证实的同倍体杂交物种形成案例只 有向日葵属植物和Heliconius属蝴蝶的 4 个杂交种。

向日葵属的Helianthus anomalus、H. deserticola 和 H. paradoxus, 是由向日葵 $(H$. annuus) 和伞花葵 (H. petiolaris)为亲本的古杂交种。这些杂交种分别 占据沙丘、沙漠和富含盐分的沼泽等环境, 而两个 广布的亲本种则很少出现在上述极端环境。Rieseberg等(2003)采用RAPD、AFLP、SSR等分子标记， 通过数量性状位点(quantitative trait locus, QTL)的 方法, 对古杂交种和人工杂交种的表现型和基因型
进行比较分析, 研究发现多数古杂交种性状差异能 够在新合成的人工杂交种中找到，且易于受到选择; 人工杂交种的极端表现型所需的亲本染色体片段 组合同样也可以在古杂交种中出现。这项研究清晰 地说明杂交有助于向日葵属植物的生态分化。

蝴蝶Heliconius heurippa的物种形成是动物中 得到很好证明的同倍体杂交起源案例(Mavárez et al, 2006; Melo et al, 2009; Salazar et al, 2010)。该物种被 认为来源于 $H$. melpomene和 $H$. cydno的杂交, 杂交 种与亲本在双翅花纹式样上存在显著差异。与花纹 式样相关的基因组区域证实了杂交的发生。通过两 个亲本之间的人工杂交所获得的后代，表现出与 $H$. heurippa相同的性状。试验观察显示，尽管杂交种 H. heurippa与亲本之间也存在微弱的基因流, 但它 们的后代倾向于与有同类花纹式样的个体交配，从 而减少了与亲本之间的基因流。研究证实杂交后的 强化作用(reinforcement)促进了杂交种与亲本的生 殖隔离。

Feliner等(2017)认同对同倍体杂交物种形成应 采取严格的生殖隔离检验，但提出不应过多关注生 殖隔离本身，还应多聚焦是否通过杂交产生丰富的 遗传创新、占据不同生境。这是因为大多数物种形 成过程中并不是完全生殖隔离的(Lowry \& Gould, 2016), 所以证明直接来自杂交而产生的生殖隔离 是不必要的。鉴于对同倍体杂交物种形成过程理解 的差异, 目前亟需对不同物种各种类型的杂交带群 体开展广泛而深入的来自形态、分子等多学科证据 的调查。不同类型的杂交带群体，包括以回交为显 著特征的杂交群，它们都是连接杂交与物种形成的 重要中间环节，保留着进化过程中重要的过渡类型， 是探讨杂交物种形成机制材料体系不可或缺的组 成部分。正是上述原因, 那些存在大量种间杂交、 曾经困扰分类学家的类群, 反过来对进化生物学家 而言, 很可能正是探寻物种形成机制与规律难得的 机遇(Dejaco et al, 2016)。

\section{6 自然杂交的进化后果和生态影响}

许多物种之间由于生境差异、空间隔离等合子 前隔离机制, 阻碍了彼此的杂交。即便可以实现种 间杂交，往往 $\mathrm{F}_{1}$ 代不育或育性极低，或面临亲本的 回交产生不育后代等合子后隔离, 无法独立成为新 种。但这种情况并不是绝对的, 如果 $F_{1}$ 代能实现单 
亲繁殖(无性繁殖或自交)、改变生殖策略(如提前或 延迟开花、吸引不同的传粉者)、占据不同的生境(生 态隔离)或者随着扩散而造成地理隔离, 就可以避 免与亲本交配, 摆脱“少数类型不利”, 最终形成新 种。大量研究表明, 种间杂交可以提供适应进化的 新材料, 以适应快速改变的环境 (Arnold, 1997; Rieseberg \& Carney, 1998)。多倍化对于促进植物物 种形成的作用已经不言而喻, 而同倍体杂交在动植 物的进化上的作用, 也越来越被认可 (Mallet, 2007)。向日葵属和Heliconius属蝴蝶的物种形成研 究为这一观点提供了强有力的支持。这些案例不仅 从形态和分子的方法证明了杂交种与亲本的关系, 观测到它们之间生殖隔离程度, 而且还通过不同的 方法证明杂交导致了这种隔离的重要作用。从目前 的研究看, 并不是涉及到同倍体杂交的物种都具备 检验是否符合Schumer等(2014b)严格生殖隔离标准 的条件, 但这应该是自然杂交与物种形成研究的重 要方向。已有的研究调查表明, 很多物种都确定为 同倍体杂交起源, 而且这种物种形成的比率很可能 被低估(Kim \& Lockhart, 2006)。例如, 通常认为大 部分动物新种的产生都是从现有物种中分化而来 的。Schwarz等(2005)的研究则显示从亚洲引种到北 美的金银花上寄生的果蝇, 来自于本土两种果蝇杂 交, 由于宿主的改变促成了种间杂交后代与亲本种 之间的生殖隔离, 导致新的同倍体杂交种产生。尽 管只发现一种通过自然杂交形成的新果蝇物种, 但 鉴于自然界中有着丰富多样的寄生动物, 通过杂交 产生新物种的方式在这类动物中很可能普遍存在。

杂交不仅仅有重要的适应性进化意义, 如促进 物种形成, 也可能带来严重的生态学问题。禾本科 多年生米草属(Spartina)植物, 因杂交而导致的生态 入侵就是典型的案例。在近一个多世纪的时间里, 该属植物经长距离的人为引种, 使其分布区超出其 原产地范围, 已经造成多次杂交事件的发生, 导致 了生态和进化上的后果, 包括生物入侵、渐渗杂交 或物种形成(Baumel et al, 2002)。这个惊人的例子显 示: 原来分布于北美东海岸的互花米草(Spartina alterniflora) 被引种到欧洲和北美西海岸, 并与当地 的土著种发生了杂交。在英国南安普敦的互花米草, 与海岸米草(S. maritima)发生杂交, 形成不育的S. $\times$ townsendii, 而在法国, 同样的亲本种杂交则形成S. $\times$ neyrautii, 它与S. $\times$ townsendii表现出相似的特性
和多倍体水平(Baumel et al, 2003)。S. × townsendii 经过染色体加倍形成了一个新的可育的、有发达根 系的异源多倍体物种一大米草(S. anglica)。由于 它能够拦截大量潮汐沉积物, 因此世界上一些国家 和地区如北欧、澳大利亚、新西兰和中国都曾有目 的地引种了大米草。但是, 这个异源多倍体能快速 入侵并扩散到周围的盐沼和河口地区, 对海岸带生 境构成潜在威胁。

非本地种入侵成功, 杂交是极为重要的遗传和 进化因素之一。杂交促进生物入侵假说(Hybridization-Invasion hypothesis, Stebbins, 1985; Abbott, 1992), 得到很多案例的支持(Ellstrand \& Schierenbeck, 2000; Schierenbeck \& Ellstrand, 2009; Hovick \& Whitney, 2014)。计算机模拟揭示即便杂交不能增 强其在本地的适应能力, 也能促进物种入侵 (Mesgaran et al, 2016)。近缘类群的渐渗杂交使得濒 危物种的保护面临现实的挑战, 如何定义杂交带来 的风险并确定相应的保护策略, 正受到越来越多的 关注和重视(Jackiw et al, 2015; Bohling, 2016)。

\section{7 研究展望}

自然杂交与物种形成的研究经历了形态、分子 标记、基因测序等常规方法鉴定, 检验相关假说, 到组学大数据的比较分析与证明, 新一代测序数据 为我们提供了杂交、多倍化与物种形成之间关系新 的见解。诸如: 在许多已经测序的物种中发现不同 程度的全基因组复制 (whole genome duplication, WGD)现象, 说明物种进化历史上的古多倍化的存 在(Jiao et al, 2011)。对六倍体(面包)小麦与近缘二倍 体的基因组测序和全基因组尺度的基因树分析揭 示了该作物驯化的历史上发生过多次杂交物种形 成(同倍体重组和多倍化)事件 (Marcussen et al, 2014); 对不同达尔文雀物种的基因组测序分析揭 示种间基因流贯穿整个适应辐射过程, 这一经典的 进化过程也有自然杂交的参与(Lamichhaney et al, 2015); 存在频繁渐渗杂交的不同种乌鸦之间, 通过 保持局部基因组的特异性来维持不同物种表型的 差异性，在基因组水平揭示渐渗杂交与物种形态的 分化的内在联系(Poelstra et al, 2014); 疮原蚊渐渗 杂交的历史可以通过系统发育基因组学的方法重 建(Fontaine et al, 2015)。

这些研究提示我们关注不同类型的自然杂交 
方式与物种形成的进化关联, 思考未来相关研究应 注意的问题, 诸如: 在基因组时代研究自然杂交, 如何对表型性状进行合理应用? 既然杂交和多倍 化是物种形成的重要方式, 为什么在一个属内很少 能鉴定到大量种间杂交? 杂交种的标准应如何界 定更为合理? 全基因组复制事件究竟和物种形成 (或分化)有何关系? 在分类学实践中, 该如何给予 种间杂交一个合理的定位? 在什么情况下, 我们应 该对种间杂交予以优先保护?

随着研究的不断深入, 关于杂交与物种形成的 概念和核心问题还会被重新讨论, 特别是生殖隔离 对于杂交的重要性、自然选择对于生殖隔离的影 响、如何定义杂交种等问题都将继续成为关注的焦 点。Payseur和Rieseberg (2016)曾提示在通过多学科 证据分析来阐释物种形成的一般规律时，应充分认 识杂交的特殊性与复杂性, 以及仅仅依靠基因组数 据作为“灵丹妙药(panacea)”来化解杂交物种形成难 题的局限性。今后, 我们开展自然杂交和物种形成 研究时, 应特别关注以下几个方面: (1)合适的研究 体系构建, 获得研究突破的往往是有很好背景的材 料, 长期研究积累了丰富的实践经验和先进的方法 设计理念, 清楚研究对象长期未解决的科学问题及 其在进化生物学上的普遍意义; (2)同倍体杂交物种 形成的群体遗传调查, 按极为严格的标准(Schumer et al, 2014b)的物种形成证明还有待深入, 需要全面 开展亲本鉴定、后代基因型分析、生殖隔离程度甚 至超亲分离的重组类型分析, 特别是比较处于物种 形成初期和不同代种间杂交的基因组与表型变化 (Nolte \& Tautz, 2010), 通过对分化谱系之间基因流 的历史重建, 分析自然选择对生殖隔离形成的影 响、物种形成过程中的生态适应变化和多样性遗传 创新的产生规律, 进而揭示杂交物种形成过程关键 环节的生物学基础; (3)中间过渡进化类型的保护, 尽管是否保护种间杂交存在争议，但更多的情况是， 由于自然发生的杂交频率较低, 以及生境的人为剧 烈扰动，很多中间过渡类型无法再次从野外获得， 如果能以合适的方式保存这些难得的资源(不包括 恶性入侵的类型), 将有助于对物种形成的不同时 期和整个过程的理解; (4)来自多学科研究成果的综 合，自然杂交与物种形成的研究涉及到形态、遗传、 生态、发育等不同过程的复杂变化, 关系到物种分 化、生殖隔离与适应不同生境等等，需探讨微观与
宏观的进化因果关系，因此亟需采用各学科的最新 方法, 开展自然杂交与物种形成的综合研究。

\section{参考文献}

Abbott RJ (1992) Plant invasions, interspecific hybridization and the evolution of new plant taxa. Trends in Ecology and Evolution, 7, 401-405.

Abbott RJ (2003) Sex, sunflowers, and speciation. Science, 301, 1189-1190.

Anderson E (1949) Introgressive Hybridization. John Wiley \& sons, New York.

Anderson E, Hubricht L (1938) Hybridization in Tradescantia. III. The evidence for introgressive hybridization. American Journal of Botany, 25, 396-402.

Anderson E, Stebbins GL Jr (1954) Hybridization as an evolutionary stimulus. Evolution, 8, 378-388.

Andrew RL, Rieseberg LH (2013) Divergence is focused on few genomic regions early in speciation: incipient speciation of sunflower ecotypes. Evolution, 67, 2468-2482.

Andrew RL, Kane NC, Baute GJ, Grassa CJ, Rieseberg LH (2013) Recent nonhybrid origin of sunflower ecotypes in a novel habitat. Molecular Ecology, 22, 799-813.

Arnold ML (1992) Natural hybridization as an evolutionary process. Annual Review of Ecology and Systematics, 85, 1893-1896.

Arnold ML (1993) Iris nelsonii: origin and genetic composition of a homoploid hybrid species. American Journal of Botany, 80, 577-583.

Arnold ML (1997) Natural Hybridization and Evolution. Oxford University Press, Oxford.

Arnold ML, Bennett BD, Zimmer EA (1990a) Natural hybridization between Iris fulva and I. hexagona: patterns of ribosomal DNA variation. Evolution, 44, 1512-1521.

Arnold ML, Hamrick JL, Bennett BD (1990b) Allozyme variation in Louisiana irises: a test for introgression and hybrid speciation. Heredity, 65, 297-306.

Arnold ML, Buckner CM, Robinson JJ (1991) Pollen mediated introgression and hybrid speciation in Louisiana irises. Proceedings of the National Academy of Sciences, USA, 188, 1398-1402.

Barb JG, Bowers JE, Renaut S, Rey JI, Knapp SJ, Rieseberg LH, Burke JM (2014) Chromosomal evolution and patterns of introgression in Helianthus. Genetics, 197, 969-979.

Baumel A, Ainouche ML, Bayer RJ, Misset MT (2002) Molecular phylogeny of hybridizing species from genus Spartina Schreb. (Poaceae). Molecular Phylogenetics and Evolution, 22, 303-314.

Baumel A, Ainouche ML, Misset MT, Gourret JP, Bayer RJ (2003) Genetic evidence for hybridization between the native Spartina maritima and the introduced Spartina alterniflora (Poaceae) in South-West France: Spartina $\times$ neyrautii re-examined. Plant Systematics and Evolution, 237, 87-97. 
Bohling JH (2016) Strategies to address the conservation threats posed by hybridization and genetic introgression. Biological Conservation, 203, 321-327.

Buerkle CA, Morris RJ, Asmussen MA, Rieseberg LH (2000) The likelihood of homoploid hybrid speciation. Heredity, 84, 441-451.

Burri R, Nater A, Kawakami T, Mugal CF, Olason PI, Smeds L, Suh A, Dutoit L, Bureš S, Garamszegi LZ, Hogner S, Moreno J, Qvarnström A, Ružić M, Sæther SA, Sætre GP, Török J, Ellegren H (2015) Linked selection and recombination rate variation drive the evolution of the genomic landscape of differentiation across the speciation continuum of Ficedula flycatchers. Genome Research, 25, 1656-1665.

Carneiro M, Albert FW, Afonso S, Pereira RJ, Burbano H, Campos R, Melo-Ferreira J, Blanco-Aguiar JA, Villafuerte R, Nachman MW, Good JM, Ferrand N (2014) The genomic architecture of population divergence between subspecies of the European rabbit. PLoS Genetics, 10, e1003519.

Chat J, Jauregui B, Petit RJ, Nadot S (2004) Reticulate evolution in kiwifruit (Actinidia, Actinidiaceae) identified by comparing their maternal and paternal phylogenies. American Journal of Botany, 91, 736-747.

Clausen J, Keck DD, Hiesey WM (1945) Experimental studies on the nature of species. II. Plant evolution through amphiploidy and autoploidy, with examples from the Madiinae. Carnegie Institution of Washington Publication, 564, 1-163.

Cui L, Wall PK, Leebens-Mack JH, Lindsay BG, Soltis DE, Doyle JJ, Soltis PS, Carlson JE, Arumuganathan K, Barakat A, Albert VA, Ma H, dePamphilis CW (2006) Widespread genome duplications throughout the history of flowering plants. Genome Research, 16, 738-749.

Dejaco T, Gassner M, Arthofer W, Schlick-Steiner BC, Steiner FM (2016) Taxonomist's nightmare ... evolutionist's delight: an integrative approach resolves species limits in jumping Bristletails despite widespread hybridization and parthenogenesis. Systematic Biology, 65, 947-974.

Duenez-Guzman EA, Mavarez J, Vose MD, Gavrilets S (2009) Case studies and mathematical models of ecological speciation. 4. Hybrid speciation in butterflies in a jungle. Evolution, 63, 2611-2626.

Eaton DAR, Ree RH (2013) Inferring phylogeny and introgression using RADseq data: an example from flowering plants (Pedicularis: Orobanchaceae). Systematic Biology, 62, 689-706.

Elder JR, Turner BJ (1995) Concerted evolution of repetitive DNA sequences in eukaryotes. Quarterly Review of Biology, 70, 297-319.

Ellegren H, Smeds L, Burri R, Olason PI, Backström N, Kawakami T, Künstner A, Mäkinen H, Nadachowska-Brzyska K, Qvarnström A, Uebbing S, Wolf JB (2012) The genomic landscape of species divergence in Ficedula flycatchers. Nature, 491, 756-760.

Ellstrand NC, Whitkus R, Rieseberg LH (1996) Distribution of spontaneous plant hybrid. Proceedings of the National Academy of Sciences, USA, 93, 5090-5093.

Ellstrand NC, Schierenbeck KA (2000) Hybridization as a stimulus for the evolution of invasiveness in plant? Proceedings of the National Academy of Sciences, USA, 97, 7043-7050.

Feliner GN, Álvarez I, Fuertes-Aguilar J, Heuertz M, Marques I, Moharrek F, Piñeiro R, Riina R, Rosselló JA, Soltis PS, Villa-Machío I (2017) Is homoploid hybrid speciation that rare? An empiricist's view. Heredity, 118, 513-516.

Ferguson D, Sang T (2001) Speciation through homoploid hybridization between allotetraploids in peonies (Paeonia). Proceedings of the National Academy of Sciences, USA, 98, 3915-3919.

Fontaine MC, Pease JB, Steele A, Waterhouse RM, Neafsey DE, Sharakhov IV, Jiang X, Hall AB, Catteruccia F, Kakani E, Mitchell SN, Wu YC, Smith HA, Love RR, Lawniczak MK, Slotman MA, Emrich SJ, Hahn MW, Besansky NJ (2015) Extensive introgression in a malaria vector species complex revealed by phylogenomics. Science, 347, 1258524.

Garrigan D, Kingan SB, Geneva AJ, Andolfatto P, Clark AG, Thornton KR, Presgraves DC (2012) Genome sequencing reveals complex speciation in the Drosophila simulans clade. Genome Research, 22, 1499-1511.

Geraldes A, Farzaneh N, Grassa CJ, McKown AD, Guy RD, Mansfield SD, Douglas CJ, Cronk QC (2014) Landscape genomics of Populus trichocarpa: the role of hybridization, limited gene flow, and natural selection in shaping patterns of population structure. Evolution, 68, 3260-3280.

Gompert Z, Lucas LK, Nice CC, Fordyce JA, Forister ML, Buerkle CA (2012) Genomic regions with a history of divergent selection affect fitness of hybrids between two butterfly species. Evolution, 66, 2167-2181.

Good JM, Vanderpool D, Keeble S, Bi K (2015) Negligible nuclear introgression despite complete mitochondrial capture between two species of chipmunks. Evolution, 69, 1961-1972.

Grant V (1981) Plant Speciation. Columbia University Press, New York.

Green RE, Krause J, Briggs AW, Maricic T, Stenzel U, Kircher M, Patterson N, Li H, Zhai W, Fritz MH, Hansen NF, Durand EY, Malaspinas AS, Jensen JD, Marques-Bonet T, Alkan C, Prüfer K, Meyer M, Burbano HA, Good JM, Schultz R, Aximu-Petri A, Butthof A, Höber B, Höffner B, Siegemund M, Weihmann A, Nusbaum C, Lander ES, Russ C, Novod N, Affourtit J, Egholm M, Verna C, Rudan P, Brajkovic D, Kucan Z, Gusic I, Doronichev VB, Golovanova LV, Lalueza-Fox C, de la Rasilla M, Fortea J, Rosas A, Schmitz RW, Johnson PL, Eichler EE, Falush D, Birney E, Mullikin JC, Slatkin M, Nielsen R, Kelso J, Lachmann M, Reich D, Pääbo S (2010) A draft sequence of the Neandertal genome. Science, 328, 710-722. 
Heliconius Genome Consortium (2012) Butterfly genome reveals promiscuous exchange of mimicry adaptations among species. Nature, 487, 94-98.

Hermansen JS, Haas F, Trier CN, Bailey RI, Nederbragt AJ, Marzal A, Sætre GP (2014) Hybrid speciation through sorting of parental incompatibilities in Italian sparrows. Molecular Ecology, 23, 5831-5842.

Hovick SM, Whitney KD (2014) Hybridisation is associated with increased fecundity and size in invasive taxa: meta-analytic support for the hybridisation-invasion hypothesis. Ecology Letters, 17, 1464-1477.

Isoda K, Shirasi S, Watanabe S, Kitamura K (2000) Molecular evidence of natural hybridization between Abies veitchii and A. homolepis (Pinaceae) revealed by chloroplast, mitochondrial and nuclear DNA markers. Molecular Ecology, 9, 1965-1974.

Jackiw RN, Mandil G, Hager HA (2015) A framework to guide the conservation of species hybrids based on ethical and ecological considerations. Conservation Biology, 29, 10401051.

Janoušek V, Wang L, Luzynski K, Dufková $\mathrm{P}$, Vyskočilová MM, Nachman MW, Munclinger P, Macholán M, Piálek J, Tucker PK (2012) Genome-wide architecture of reproductive isolation in a naturally occurring hybrid zone between Mus musculus musculus and M. m. domesticus. Molecular Ecology, 21, 3032-3047.

Janoušek V, Munclinger P, Wang L, Teeter KC, Tucker PK (2015) Functional organization of the genome may shape the species boundary in the house mouse. Molecular Biology and Evolution, 32, 1208-1220.

Jiao Y, Wickett NJ, Ayyampalayam S, Chanderbali AS, Landherr L, Ralph PE, Tomsho LP, Hu Y, Liang H, Soltis PS, Soltis DE, Clifton SW, Schlarbaum SE, Schuster SC, Ma H, Leebens-Mack J, dePamphilis CW (2011) Ancestral polyploidy in seed plants and angiosperms. Nature, 473, 97-100.

Jónsson H, Schubert M, Seguin-Orlando A, Ginolhac A, Petersen L, Fumagalli M, Albrechtsen A, Petersen B, Korneliussen TS, Vilstrup JT, Lear T, Myka JL, Lundquist J, Miller DC, Alfarhan AH, Alquraishi SA, Al-Rasheid KA, Stagegaard J, Strauss G, Bertelsen MF, Sicheritz-Ponten T, Antczak DF, Bailey E, Nielsen R, Willerslev E, Orlando L (2014) Speciation with gene flow in equids despite extensive chromosomal plasticity. Proceedings of the National Academy of Sciences, USA, 111, 18655-18660.

Keller I, Wagner CE, Greuter L, Mwaiko S, Selz OM, Sivasundar A, Wittwer S, Seehausen O (2013) Population genomic signatures of divergent adaptation, gene flow and hybrid speciation in the rapid radiation of Lake Victoria cichlid fishes. Molecular Ecology, 22, 2848-2863.

Kim MB, Lockhart PJ (2006) Reconstructing reticulate evolutionary histories of plants. Trends in Plant Science, 11, 398-404.

Kim ST, Sultan SE, Donoghue MJ (2008) Allopolyploid speci- ation in Persicaria (Polygonaceae): insights from a lowcopy nuclear region. Proceedings of the National Academy of Sciences, USA, 105, 12370-12375.

Lamichhaney S, Berglund J, Almén MS, Maqbool K, Grabherr M, Martinez-Barrio A, Promerová M, Rubin CJ, Wang C, Zamani N, Grant BR, Grant PR, Webster MT, Andersson L (2015) Evolution of Darwin's finches and their beaks revealed by genome sequencing. Nature, 518, 371-375.

Lee JF, Mummenhoff K, Bowman JL (2002) Allopolyploidization and evolution of species with reduced floral structures in Lepidium L. (Brassicaceae). Proceedings of the National Academy of Sciences, USA, 99, 16835-16840.

Lewontin RC, Birch LC (1966) Hybridization as a source of variation for adaptation to new environments. Evolution, 20, 315-336.

Liu KJ, Steinberg E, Yozzo A, Song Y, Kohn MH, Nakhleh L (2015) Interspecific introgressive origin of genomic diversity in the house mouse. Proceedings of the National Academy of Sciences, USA, 112, 196-201.

Lohse K, Clarke M, Ritchie MG, Etges WJ (2015) Genome-wide tests for introgression between cactophilic Drosophila implicate a role of inversions during speciation. Evolution, 69, 1178-1190.

Lohse K, Frantz LA (2014) Neandertal admixture in Eurasis confirmed by maximum-likelihood analysis of three genomes. Genetics, 196, 1241-1251.

Lotsy JP (1916) Evolution by Means of Hybridization. Martinus Nijhoff, The Hague.

Lowry DB, Gould BA (2016) Speciation continuum. In: Encyclopedia of Evolutionary Biology (ed. Kliman R), pp. 159-165. Academic Press, Oxford, UK.

Mallet J (2005) Hybridization as an invasion of the genome. Trends in Ecology and Evolution, 20, 229-237.

Mallet J (2007) Hybrid speciation. Nature, 446, 279-283.

Marcussen T, Sandve SR, Heier L, Spannagl M, Pfeifer M, International Wheat Genome Sequencing Consortium, Jakobsen KS, Wulff BB, Steuernagel B, Mayer KF, Olsen OA (2014) Ancient hybridizations among the ancestral genomes of bread wheat. Science, 345, 1250092.

Martin SH, Dasmahapatra KK, Nadeau NJ, Salazar C, Walters JR, Simpson F, Blaxter M, Manica A, Mallet J, Jiggins CD (2013) Genome-wide evidence for speciation with gene flow in Heliconius butterflies. Genome Research, 23, 1817-1828.

Martin CH, Cutler JS, Friel JP, Dening Touokong C, Coop G, Wainwright PC (2015) Complex histories of repeated gene flow in Cameroon crater lake cichlids cast doubt on one of the clearest examples of sympatric speciation. Evolution, 69, $1406-1422$.

Masterson J (1994) Stomatal size in fossil plants: evidence for polyploidy in majority of angiosperms. Science, 264, 421424.

Mavárez J, Salazar CA, Bermingham E, Salcedo C, Jiggins CD, Linares M (2006) Speciation by hybridization in Heliconius 
butterflies. Nature, 441, 868-871.

Mayr E (1992) A local flora and the biological species concept. American Journal of Botany, 79, 222-238.

McCarthy EM, Asmussen MA, Anderson WW (1995) A theoretical assessment of recombinational speciation. Heredity, 74, 502-509.

Meier JI, Marques DA, Mwaiko S, Wagner CE, Excoffier L, Seehausen O (2017) Ancient hybridization fuels rapid cichilid fish adaptive radiations. Nature Communications, 8 , 14363.

Melo MC, Salazar C, Jiggins CD, Linares M (2009) Assortative mating preferences among hybrids offers a route to hybrid speciation. Evolution, 63, 1660-1665.

Mesgaran MB, Lewis MA, Ades PK, Donohue K, Ohadi S, Li C, Cousens RD (2016) Hybridization can facilitate species invasions, even without enhancing local adaptation. Proceedings of the National Academy of Sciences, USA, 113, 10210-10214.

Mogensen HL (1996) The hows and whys of cytoplasmic inheritance. American Journal of Botany, 83, 383-404.

Morin PA, Archer FI, Foote AD, Vilstrup J, Allen EE, Wade P, Durban J, Parsons K, Pitman R, Li L, Bouffard P, Abel Nielsen SC, Rasmussen M, Willerslev E, Gilbert MT, Harkins T (2010) Complete mitochondrial genome phylogeographic analysis of killer whales (Orcinus orca) indicates multiple species. Genome Research, 20, 908-916.

Nadachowska-Brzyska K, Burri R, Olason PI, Kawakami T, Smeds L, Ellegren H (2013) Demographic divergence history of pied flycatcher and collared flycatcher inferred from whole-genome re-sequencing data. PLoS Genetics, 9, e1003942.

Nadeau NJ, Martin SH, Kozak KM, Salazar C, Dasmahapatra KK, Davey JW, Baxter SW, Blaxter ML, Mallet J, Jiggins CD (2013) Genome-wide patterns of divergence and gene flow across a butterfly radiation. Molecular Ecology, 22, 814-826.

Nolte AW, Tautz D (2010) Understanding the onset of hybrid speciation. Trends in Genetics, 26, 54-58.

O'Loughlin SM, Magesa S, Mbogo C, Mosha F, Midega J, Lomas S, Burt A (2014) Genomic analyses of three malaria vectors reveals extensive shared polymorphism but contrasting population histories. Molecular Biology and Evolution, 31, 889-902.

Otto SP, Whitton J (2000) Polyploid incidence and evolution. Annual Review of Genomics, 34, 401-437.

Parchman TL, Gompert Z, Braun MJ, Brumfield RT, McDonald DB, Uy JA, Zhang G, Jarvis ED, Schlinger BA, Buerkle CA (2013) The genomic consequences of adaptive divergence and reproductive isolation between species of manakins. Molecular Ecology, 22, 3304-3317.

Payseur BA, Rieseberg LH (2016) A genomic perspective on hybridization and speciation. Molecular Ecology, 25, 23372360 .
Phifer-Rixey M, Bomhoff M, Nachman MW (2014) Genome-wide patterns of differentiation among house mouse subspecies. Genetics, 198, 283-297.

Poelstra JW, Vijay N, Bossu CM, Lantz H, Ryll B, Müller I, Baglione V, Unneberg P, Wikelski M, Grabherr MG, Wolf JBW (2014) The genomic landscape underlying phenotypic integrity in the face of gene flow in crows. Science, 344, 1410-1414.

Prüfer K, Racimo F, Patterson N, Jay F, Sankararaman S, Sawyer S, Heinze A, Renaud G, Sudmant PH, de Filippo C, Li H, Mallick S, Dannemann M, Fu Q, Kircher M, Kuhlwilm M, Lachmann M, Meyer M, Ongyerth M, Siebauer M, Theunert C, Tandon A, Moorjani P, Pickrell J, Mullikin JC, Vohr SH, Green RE, Hellmann I, Johnson PL, Blanche H, Cann H, Kitzman JO, Shendure J, Eichler EE, Lein ES, Bakken TE, Golovanova LV, Doronichev VB, Shunkov MV, Derevianko AP, Viola B, Slatkin M, Reich D, Kelso J, Pääbo S (2014) The complete genome sequence of a Neanderthal from the Altai Mountains. Nature, 505, 43-49.

Ramsey J, Schemske DW (2002) Neopolyploidy in flowering plants. Annual Review of Ecology and Systematics, 33, 589-639.

Renaut S, Grassa CJ, Yeaman S, Moyers BT, Lai Z, Kane NC, Bowers JE, Burke JM, Rieseberg LH (2013) Genomic islands of divergence are not affected by geography of speciation in sunflowers. Nature Communications, 4, 1827.

Rheindt FE, Fujita MK, Wilton PR, Edwards SV (2014) Introgression and phenotypic assimilation in Zimmerius flycatchers (Tyrannidae): population genetic and phylogenetic inferences from genome-wide SNPs. Systematic Biology, 63, 134-152.

Rieseberg LH (1991) Homoploid reticulate evolution in Helianthus (Asteraceae): evidence from ribosomal genes. American Journal of Botany, 78, 1218-1237.

Rieseberg LH, Carney SE (1998) Tansley review No. 102: Plant hybridization. New Phytologist, 140, 599-624.

Rieseberg LH, Carter R, Zona S (1990) Molecular tests of the hypothesized hybrid origin of two diploid Helianthus species (Asteraceae). Evolution, 44, 1498-1511.

Rieseberg LH, Raymond O, Rosenthal DM, Lai Z, Livingstone K, Nakazato T, Durphy JL, Schwarzbach AE, Donovan LA, Lexer C (2003) Major ecological transitions in wild sunflowers facilitated by hybridization. Science, 301, 1211-1216.

Rieseberg LH, Wendel JF (1993) Introgression and its consequences in plants. In: Hybrid Zones and the Evolutionary Process (ed. Harrison RG), pp. 70-109. Oxford University Press, Oxford.

Russell A, Samuel R, Klejna V, Barfuss MH, Rupp B, Chase MW (2010) Reticulate evolution in diploid and tetraploid species of Polystachya (Orchidaceae) as shown by plastid DNA sequences and low-copy nuclear genes. Annals of Botany, 106, 37-56.

Salazar C, Baxter SW, Pardo-Diaz C, Wu G, Surridge A, 
Linares M, Bermingham E, Jiggins CD (2010) Genetic evidence for hybrid trait speciation in Heliconius butterflies. PLoS Genetics, 6, 1-12.

Sang T, Crawford DJ, Stuessy TF (1995) Documentation of reticulate evolution in peonies (Paeonia) using internal transcribed spacer sequences of nuclear ribosomal DNA: implications from biogeography and concerted evolution. Proceedings of the National Academy of Sciences, USA, 92, 6813-6817.

Sang T, Donoghue MJ, Zhang D (1997) Evolution of alcohol dehydrogenase genes in peonies (Paeonia): phylogenetic relationships of putative nonhybrid species. Molecular Biology and Evolution, 14, 994-1007.

Sankararaman S, Patterson N, Li H, Pääbo S, Reich D (2012) The date of interbreeding between Neandertals and modern humans. PLoS Genetics, 8, e1002947.

Sankararaman S, Mallick S, Dannemann M, Prüfer K, Kelso J, Pääbo S, Patterson N, Reich D (2014) The genomic landscape of Neanderthal ancestry in present-day humans. Nature, 507, 354-357.

Schemske DW (2000) Understanding the origin of species. Evolution, 54, 1069-1073.

Schierenbeck KA, Ellstrand NC (2009) Hybridization and the evolution of invasiveness in plants and other organisms. Biological Invasions, 11, 1093-1105.

Schumer M, Cui R, Powell DL, Dresner R, Rosenthal GG, Andolfatto P (2014a) High-resolution mapping reveals hundreds of genetic incompatibilities in hybridizing fish species. eLife, 3, e02535.

Schumer M, Cui R, Rosenthal GG, Andolfatto P (2015) Reproductive isolation of hybrid populations driven by genetic incompatibilities. PLoS Genetics, 11, e1005041.

Schumer M, Rosenthal GG, Andolfatto P (2014b) How common is homoploid hybrid speciation? Evolution, 68, 1553-1560.

Schwarz D, Matta BM, Shakir-Botteri NL, McPheron BA (2005) Host shifts to an invasive plant triggers rapid animal hybrid speciation. Nature, 436, 546-549.

Smedmark JE, Eriksson T, Evans RC, Campbell CS (2003) Ancient allopolyploid speciation in Geinae (Rosaceae): evidence from nuclear granule-bound starch synthase (GBSSI) gene sequences. Systematic Biology, 52, 374-385.

Soltis DE, Soltis PS (1993) Molecular data and the dynamic nature of polyploidy. Critical Reviews in Plant Sciences, 12, 243-273.

Stace CA (1975) Introductory. In: Hybridization and Flora of the British Isles (ed. Stace CA), pp. 1-90. Academic Press, London.

Stebbins GL Jr (1947) Types of polyploids: their classification and significance. Advances in Genetics, 1, 403-429.

Stebbins GL Jr (1985) Polyploidy, hybridization, and the invasion of new habitats. Annals of the Missouri Botanical Garden, 72, 824-832.

Stölting KN, Nipper R, Lindtke D, Caseys C, Waeber S, Castiglione S, Lexer C (2013) Genomic scan for single nucleotide polymorphisms reveals patterns of divergence and gene flow between ecologically divergent species. Molecular Ecology, 22, 842-855.

Streicher JW, Devitt TJ, Goldberg CS, Malone JH, Blackmon H, Fujita MK (2014) Diversification and asymmetrical gene flow across time and space: lineage sorting and hybridization in polytypic barking frogs. Molecular Ecology, 23, 3273-3291.

Vriesendorp B, Bakker FT (2005) Reconstructing patterns of reticulate evolution in angiosperms: what can we do? Taxon, 54, 593-604.

Wagner WH Jr (1970) Biosystematics and evolutionary noise. Taxon, 19, 146-151.

Wall JD, Yang MA, Jay F, Kim SK, Durand EY, Stevison LS, Gignoux C, Woerner A, Hammer MF, Slatkin M (2013) Higher levels of Neanderthal ancestry in East Asians than in Europeans. Genetics, 194, 199-209.

Wang L, Luzynski K, Pool JE, Janoušek V, Dufková P, Vyskočilová MM, Teeter KC, Nachman MW, Munclinger P, Macholán M, Piálek J, Tucker PK (2011) Measures of linkage disequilibrium among neighbouring SNPs indicate asymmetries across the house mouse hybrid zone. Molecular Ecology, 20, 2985-3000.

Wang XR, Szmidt AE, Savolainen O (2001) Genetic composition and diploid hybrid speciation of a high mountain pine, Pinus densata, native to the Tibetan Plateau. Genetics, 159, 337-346.

Wendel JF, Doyle JJ (1998) Phylogenetic incongruence: window into genome history and molecular evolution. In: Molecular Systematics of Plants. II. DNA Sequencing (eds Soltis DE, Soltis PS, Doyle JJ), pp. 265-296. Kluwer Academic Publishers, Boston/Dordrecht/London.

Wendel JF, Schnabel A, Seelanan T (1995) An unusual ribosomal DNA sequence from Gossypium gossypioides reveals ancient, cryptic, intergenomic introgression. Molecular Phylogenetics and Evolution, 4, 298-313.

Wood TE, Takebayashi N, Barker MS, Mayrose I, Greenspoon PB, Rieseberg LH (2009) The frequency of polyploid speciation in vascular plants. Proceedings of the National Academy of Sciences, USA, 106, 13875-13879.

(责任编委: 葛学军 责任编辑: 问文杰) 